\title{
AIDS Clinical Trials
}

\section{Is There Access for All?}

I

$\mathrm{n}$ this issue of JGIM, Stone and colleagues have asked an important question. ${ }^{1}$ Among patients with HIV infection, do persons of color, women, and drug users participate in clinical trials for new treatments of their disease as readily as do white men who do not use drugs? Patients at the authors' institution should have ready access to clinical trials sponsored by the National Institute of Allergy and Infectious Diseases through an on-site AIDS Clinical Trials Unit (ACTU). But patients of color and drug users were less likely to participate in clinical trials, and patients of color and women were less likely to consider themselves informed about clinical trial opportunities. These authors are not the first to find a difference in participation in AIDS clinical trials based on race. In 1995, Diaz et al. also found that among adults with AIDS, blacks were less likely than other adults with AIDS to participate in clinical trials, as were those with less than a high school education and those who did not have a regular source of health care. ${ }^{2}$ Why should we be troubled by these differing rates of participation, and what can we do to understand them better?

Lack of participation in HIV clinical trials by persons of color, women, and drug users should trouble us deeply for three principal reasons. First, changes in surrogate markers, such as CD4 lymphocyte count and quantitative assessment of viral load, are used instead of clinical outcomes to expedite approval of antiretroviral therapies and in initiating, monitoring, and changing these therapies. ${ }^{3,4}$ It may not, however, be correct to assume that changes in surrogate markers, which are used to project the impact of a treatment on the progression of HIV disease, are similar in different racial groups, in different risk groups, and in men and women. ${ }^{5}$ Only by enrolling adequate numbers of persons of color, injection drug users, and women will we fully understand how to interpret changes in surrogate markers in these groups.

Second, it is not always correct to assume that drugs will behave similarly in different racial groups, or in men and women. ${ }^{6-8}$ Knowledge of differences in clinical response to medications and potential toxicities that may exist between different racial, ethnic, and gender groups is essential for physicians providing care to these patients.

Third, it has been noted (without full explanation) that throughout the United States, persons of color have less access to health care and lower utilization of health services than do whites. ${ }^{9-12}$ As noted by Stone et al., lack of participation in HIV clinical trials by minorities, women, and injection drug users means lack of access to the newest treatments for the disease. Witness the startling advances in the treatment of AIDS with the development of protease inhibitors. ${ }^{13-15}$ These drugs have been available by prescription only since late 1995, but before that they were available through clinical trials.

In the Stone study, the reasons given most often for not participating in clinical trials were: not being informed about the trials; a lack of interest; and (less often) fear of experimentation. Latino patients were twice as likely as others to report being uninformed. As the authors note, the generalizability of this study may be limited. The demographics of this group of patients differ from those of many communities in race and ethnicity, route of HIV acquisition, and socioeconomic status. But perhaps more importantly, we must recognize that participation by patients in clinical trials depends as much on the physicians, nurses, and other personnel who staff clinical trials units as it does on the patients.

We are told little about the staff in the present study. For many clinical trials, whether sponsored by government or by industry, patients do not self-refer, but are recruited, sometimes aggressively. Researchers review patient charts to identify those who are eligible for a clinical trial. A patient's primary care provider may be approached and asked if the patient would make "a good study candidate," meaning someone who is likely to keep most appointments, someone who is judged to be at least fairly responsible and somewhat reliable, someone who is not suffering from a coexisting mental illness. There may also be an unrecognized tendency by those recruiting to overlook patients who have chaotic life styles, including women with small children, for whom keeping appointments may be complicated. Patients thought to have a substance abuse problem are less likely to be perceived as good study candidates.

Communicating details about trial participation with patients who are not fluent in English may be difficult, and researchers may incorrectly infer a lack of interest or understanding. ${ }^{16}$ Whether such judgments are made knowingly or otherwise, we cannot fully understand the differing rates of participation by persons of color, women, and drug users until we learn more about the role of staff judgment on subject recruitment.

Nonetheless, Stone and colleagues have underscored that a problem exists at their institution and likely exists in many other academic medical centers. We need not wait for complete information to begin responding. The authors advise us that, as a profession, we need to develop greater cultural competence in discussing clinical trials with patients from a diversity of backgrounds. We need to develop appropriate educational material about clinical trials for patients of color. We need to overcome language barriers. In addition, incentives can be devised 
that will appeal to under-represented populations. The research coordinator in my ACTU has had remarkable success in recruiting women and persons of color by offering baby-sitting for young children, plus public transportation tokens and meal vouchers for the study participant and any family members or friends who accompany the patient for study visits. (Debora Dunbar, personal communication, December 30, 1996.) At a more fundamental level, we need to continue efforts to help students and residents communicate more effectively with a wide array of patients on a broad spectrum of issues. A necessary component of this effort is to help trainees understand more about different cultures and the health beliefs that are part of these cultures.

Stone et al. do not report the reasons given for trial participation among the $22 \%$ of patients who took part in a study, and this information could be helpful in learning how to increase participation among persons of similar backgrounds. The very advances in the treatment of HIV infection that have brought an atmosphere of heightened hope may make it more difficult to recruit patients for additional trials. The ready availability of combination therapy using protease inhibitors and reverse transcriptase inhibitors may make patients less likely to self-refer, and doctors less likely to recommend trial participation. For this reason, and because it is equitable and just, doctors caring for patients with HIV infection should learn to discuss relevant trials with all patients who are medically appropriate candidates, just as we refer all patients for preventive health studies. The research team must also communicate that every effort will be made to help every patient succeed in a trial.

Race, gender, and risk factors will continue to affect the clinical treatment of patients with HIV infection, but they must stop being barriers to participation in clinical trials. The work of Stone and her colleagues serves to make us aware of the problem of differential participation in clinical trials. More work to understand the problem, particularly the role of health care providers, is needed before we can expect significant change.-LORNA A. LYNN, MD, University of Pennsylvania, Philadelphia.

\section{REFERENCES}

1. Stone VE, Mauch MY, Steger K, Janas SF, Craven DE. Race, gender, drug use and participation in AIDS clinical trials: lessons from a municipal hospital cohort. J Gen Intern Med. 1997;12:150-7.

2. Diaz T, Chu SY, Sorvillo F, et al. Differences in participation in experimental drug trials among persons with AIDS. Journal of Acquired Immune Deficiency Syndromes and Human Retrovirology. 1995; 10:562-8.

3. Lagakos SW. Surrogate markers in AIDS clinical trials: conceptual basis, validation, and uncertainties. Clin Infect Dis. 1993;16(Suppl 1):S22-5.

4. Katzenstein DA, Holodniy M. HIV viral load quantification, HIV resistance, and antiretroviral therapy. AIDS Clin Rev. 1995;277-303.

5. Gorter RW, Vranizan KM, Osmond DH, Moss AR. Differences in laboratory values in HIV infection by sex, race, and risk group. AIDS. 1992;6:1341-7.

6. Stretcher BN. Pharmacokinetic optimisation of antiretroviral therapy in patients with HIV infection. Clin Pharmacokinet. 1995;20: $46-65$.

7. Blantz RC. Acetaminophen: acute and chronic effects on renal function. Am J Kidney Dis. 1996;28:S3-6.

8. Yonkers KA, Kando JC, Cole JO, Blumenthal S. Gender differences in pharmacokinetics and pharmacodynamics of psychotropic medication. Am J Psychiatry. 1992;149:587-95.

9. Phillips RS, Hamel MB, Teno JM, et al. Race, resource use, and survival in seriously ill hospitalized adults. J Gen Intern Med. 1996;11:387-96.

10. Mort EA, Weissman JS, Epstein AM. Physician discretion and racial variation in the use of surgical procedures. Arch Intern Med. 1994;154:761-7.

11. Goldberg KC, Hartz AJ, Jacobsen SJ, Krakauer H, Rimm AA. Racial and community factors influencing coronary artery bypass graft surgery rates for all 1986 Medicare patients. JAMA. 1992; 267:1473-7.

12. Lieu TA, Newacheck PW, McManus MA. Race, ethnicity, and access to ambulatory care among US adolescents. Am J Public Health. 1993;83:960-5.

13. Collier AC, et al. Treatment of human immunodeficiency virus infection with saquinavir, zidovudine, and zalcitabine. N Engl J Med 1996:334:1011-7.

14. Markowitz M, Saag M, Powderly WE, et al. A preliminary study of ritonavir, an inhibitor of HIV-1 protease, to treat HIV-1 infection. N Engl J Med. 1995;333:1534-9.

15. Carpenter CC, Fischl MA, Hammer SM, et al. Antiretroviral therapy for HIV infection in 1996; recommendations of an international panel. JAMA. 1996;276:146-54.

16. Hazuda HP. Non-English-speaking patients: a challenge to researchers. J Gen Intern Med. 1996;11:56-7. 